\title{
Propionibacterium acnes
}

Das Ziel der prospektiven Studie war es, die Häufigkeit der Besiedlung mit Propionibacterium acnes bei Patienten mit primärer Schulterendoprothetik und patientenbezogene Risikofaktoren zu identifizieren, sowie die Wirksamkeit des perioperativen aseptischen Regimes mit Cefazolin-Antibiotikumprophylaxe zu bewerten.

Koh CK, Marsh JP, Drinković D et al. Propionibacterium acnes in primary shoulder arthroplasty: rates of colonization, patient risk factors, and efficacy of perioperative prophylaxis. J Shoulder Elbow Surg 2015; DOI: 10.1016/j.jse.2015.09.033

In diese Studie wurden in einem neuseeländischen Krankenhaus 30 aufeinanderfolgende Patienten eingeschlossen, die eine primäre Schulterendoprothese erhalten haben und ohne vorgegangene Schulteroperation waren. Es wurde:

1. eine sehr hohe Inzidenz einer Besiedlung mit Propionibacterium acnes (P. acnes) gezeigt

2. das männliche Geschlecht und das Vorhandensein von Haaren als Risikofaktoren identifziert

3. die mangelhafte Wirksamkeit einer perioperativen antibiotischen Prophylaxe mittels Cefazolin gezeigt.

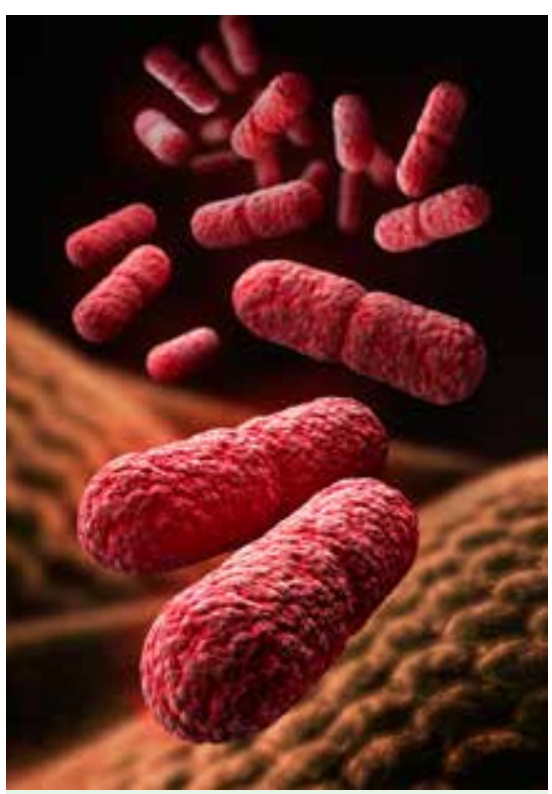

Propionibacterium acnes Bakterien sind grampositive Stäbchen und Bestandteil der normalen Hautflora (Bild: psdesign1 / Fotolia.com).

\section{Hintergrund \\ $\nabla$}

Das P. acnes ist ein gram-positiver anaerober Erreger mit Vorliebe zur Besiedlung in der Schulterregion. Dieses Bakterium wurde als Erreger von postoperativen Schulterinfektionen identifiziert. Die Diagnosestellung einer Infektion mit P. acnes kann aufgrund des indolenten Verlaufes und der minimalen Wirkung auf biochemische Marker problematisch sein. Diesen Erreger mit dem routinemäßigen aseptischen perioperativen Vorgehen um eine Operation zu eradizieren, gelingt in vielen Fällen nicht, da er in den Talgdrüsen der Haarfollikel beheimatet ist. Aktuelle Studien haben positive Kulturen von P. acnes in etwa $40 \%$ der Patienten bei primärer Schulter- oder Revisionsschulterprothetik nachgewiesen.

\section{Methodik \\ $\nabla$}

Es wurden perioperativ 6 Abstriche, die in 4 oberflächliche Haut- und 2 tiefe Wundabstriche aufgeteilt waren, genommen. Anschließend erfolgte eine quantitative Analyse der bakteriellen Besiedlung mit P. acnes. Des Weiteren wurde die minimale Hemmkonzentration von Cefazolin für P. acnes aus den positiven Kulturen bestimmt.

\section{Ergebnisse}

Bei 22 Patienten (73\%) wurde P. acnes positiv nachgewiesen. Das männliche Geschlecht $(\mathrm{p}=0,024)$ und das Vorhandensein von Haaren $(p=0,005)$ zeigten sich als
Risikofaktoren: es wurden eine signifikant höhere Rate von Besiedlungen bei den oberflächlichen Abstrichen nachgewiesen. Die Patienten mit einem positivem Nachweis bei den oberflächlichen Abstrichen ( $p=0,076)$ und mit Vorhandensein von Haaren in Kombination mit Steroidinjektion in der Vorgeschichte zeigten zwar häufiger einen positiven Nachweis von P. acnes bei den tiefen Abstrichen, jedoch war dies nicht statistisch signifikant. Die lokalen topischen antiseptischen Maßnahmen und die Cefazolinprophylaxe führten nicht zur einer quantitativen Reduktion von P. acnes ( $\mathrm{p}=0,12)$. Die minimale Hemmkonzentration von Cefazolin für P. acnes betrug $0,32 \mu \mathrm{g} / \mathrm{ml}$.

\section{Kommentar}

$\nabla$

Es handelt um eine valide Studie, die die Kolonisationsraten, die möglichen Risikofaktoren, sowie die minimale Hemmkonzentration von Cefazolin aufzeigt. Kritisch zu betrachten sind mögliche Risikofaktoren, die in dieser Studie nicht betrachtet wurden (z. B. Diabetes mellitus, Adipositas, etc.), die jedoch ebenfalls eine Rolle bei der Kolonisation mit P. acnes spielen könnten. Des Weiteren hätte die lokale Konzentration von Cefazolin bestimmt werden können, um Aussagen über die lokale Wirkung zu machen. Unklar bleibt auch die klinische Bedeutung der Besiedlung mit P. acnes und ihr quantitativer $\mathrm{Zu}-$ sammenhang mit einer möglichen späteren Infektion. Die Studie zeigt deutlich die Insuffizienz des bisherigen perioperativen Regimes, zeigt jedoch leider keine wirksameren Alternativen auf.

Dr. med. Marc-Frederic Pastor Orthopädische Klinik der Medizinischen Hochschule Hannover marc-frederic.pastor@diakovere.de 